\title{
Determining host genetic susceptibility or resistance to bovine digital dermatitis in cattle
}

\section{R A Scholey ${ }^{1,2}$, W E R Ollier ${ }^{2}$, R W Blowey ${ }^{3}$, R D Murray ${ }^{1}$, S D Carter ${ }^{1}$}

${ }^{1}$ University of Liverpool, Liverpool, United Kingdom, ${ }^{2}$ University of Manchester, Manchester, United Kingdom, ${ }^{3}$ Wood Veterinary Group, Gloucester, United Kingdom

Email:rscholey@liv.ac.uk

Introduction Bovine digital dermatitis (BDD) is a bacterial infection of the hoof, causing painful lesion formation and progressive lameness. Treponemes have been implicated as the causative organism [1]. Primarily affecting commercial dairy herds, the disease presents agriculture with a huge economic and animal welfare problem. Within any herd there appears to be great variation in the way cattle are affected; some are recurrently and severely affected, where as others are relatively untouched by digital dermatitis, suggesting an underlying genetic susceptibility. Using a candidate gene approach we are investigating whether there is any genetic pre-disposition to the disease, by examining variations in the DNA known as single nucleotide polymorphisms (SNPs), in key genes with immune function. This study examines what SNP differences exist in BDD affected cattle, BDD unaffected cattle and in different breeds of cattle with differing BDD susceptibilities. Alongside this, we have conducted a genome wide association study using the Illumina bovine SNP-50 bead chip, to investigate SNP differences across the genome of BDD affected and BDD unaffected animals. Ultimately this study aims to identify key SNPs that may be associated with susceptibility or resistance to bacterial treponeme infection, and thus development of digital dermatitis, in cattle.

Materials and methods Cattle from three commercial pedigree Holstein Friesian farms were screened over a period of several months to identify BDD affected and BDD un-affected animals. DNA samples were obtained from BDD affected cattle $(\mathrm{n}=88)$ and BDD unaffected cattle $(\mathrm{n}=114)$. In addition DNA samples were collected from eight various other breeds of beef and dairy cattle (total $\mathrm{n}=178$ ). Fifteen immune-related candidate genes were identified by literature search including CD14, iCAM1, IL1B, IL6, S10A8, MBL, VDR, iNOS, NRAMP1, TNFa, TIMP2, MMP9, TLR4, TLR2, IFN- $\gamma$. In this group of genes there were 60 publicly available SNPs that were of interest to the study. Eleven candidate genes had few SNPs available so SNP discovery was undertaken. The DNA of 10 Holstein-Friesian, 10 Guernsey, and 7 British Whites was screened for novel SNPs using Transgenomic WAVE technology. The screened fragments yielded 70 novel SNPs. Genotyping assays (Sequenom mass-array technology) were designed to validate the panel of novel and public SNPs, and determine allele frequencies in the cohort of BDD unaffected animals, BDD affected animals and in the different cattle breeds. In addition the Illumina bovine SNP-50 bead chip was used to screen DNA samples from 24 of the BDD affected animals and 24 of the BDD unaffected animals.

Results A preliminary analysis of the genotyping data suggests several of the candidate gene SNPs are significantly associated with BDD susceptibility/resistance. A preliminary analysis of the whole genome screening with the illumina SNP-50 bead chip suggests there are several regions on the genome where SNPs are associated with BDD resistance/susceptibility. Figure 1 shows a plot of the significance of SNP associations after population stratification was taken into account and out liars removed for the BDD affected group $(n=19)$ and the BDD unaffected group $(n=21)$. The most associated SNP (chromosome 26) had a p value of 2.23E-5 (corrected using 1000 permutations). Currently a confirmation study is being performed on the most associated SNPs in a larger cohort of affected and unaffected animals.

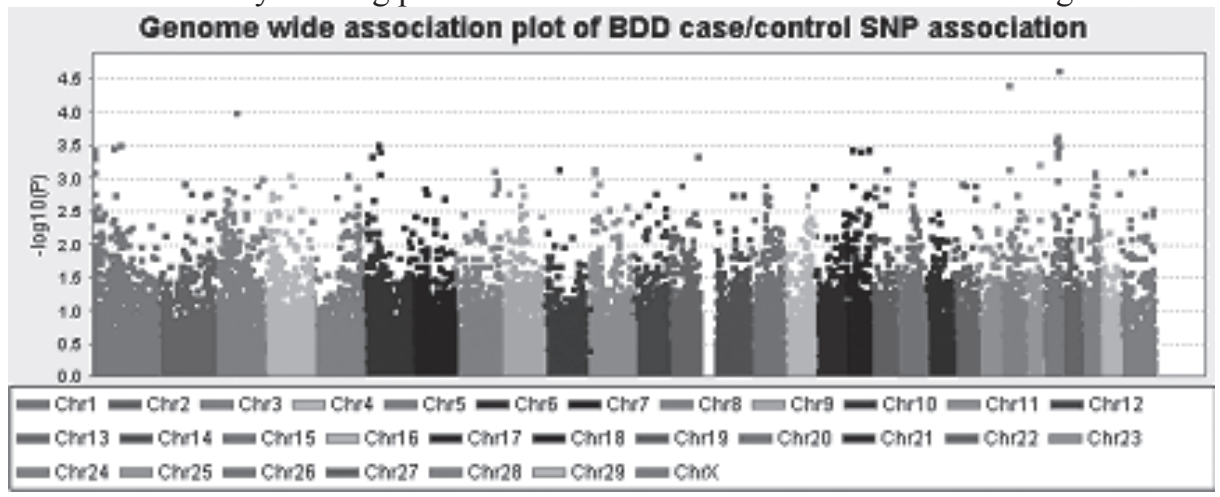

\section{Figure 1}

Conclusion This study has found some potentially interesting SNPs that are associated with susceptibility/resistance to $\mathrm{BDD}$ and therefore also bacterial treponeme infection. Once susceptibility/resistance SNPs are identified, populations can be screened for alleles associated with the disease. The results may help reduce digital dermatitis in dairy cattle by better informing breeding strategies. The principles of this project can be applied to a range of cattle infectious diseases, and will be useful in addressing the ever increasing consumer demands for improved animal welfare and food safety.

Acknowledgements This work is funded by the veterinary training research initiative (VTRI) scheme and the BBSRC.

\section{Reference}

Demirkan I., Carter S.D., Murray R.D., Blowey R.W. and Woodward M.J. 1998. The frequent detection of a treponeme in bovine digital dermatitis by immunocytochemistry and polymerase chain reaction. Veterinary Microbiology 60, $285-292$. 